\title{
Implementation of Playout Algorithm to Streaming Media File for Reducing Packet Loss Over Internet Protocol
}

\author{
Kasman,* Shung Ping Chen, Johannes Gerdes, and Moch. Hariadi \\ Electrical Engineering Department, \\ Technology Institute of Sepuluh Nopember \\ ITS Campus, Sukolilo, Surabaya 60111
}

\begin{abstract}
PlayOut Algorithm has considerable contributed to the interactive communication over Internet like voice communication and VoIP. Some papers have, including playout adaptive Algorithm, investigated how to reduce and minimize the effect of Jitter which are much constitute to the packet loss. In streaming media file using H.323 format for video has been investigated in this paper. By using Network Analyzer for various network condition has represented network behaviour over the network VLAN. The use of playout Algorithm to estimate the buffering delay time for scheduling each packet arrived in receiver has remained the reduction of packet loss under $5 \%$ for traffic load utilization generator up to $21 \%$ using Network Analyzer.
\end{abstract}

KEYWORDS: playout algorithm, buffering delay, delay, jitter, packet loss, VLAN

\section{INTRODUCTION}

The using streaming file for real time transmission for video and audio through internet is a very much difficult to playout the received data at the receiver perfectly because of delay, variation delay (jitter) and loss of packet [1]. A typical delay and loss can not be guaranteed due to some parameters of the network and the distance between sender and receiver sites. But the values considered acceptable for the packet loss and latency are $0-20 \%$ and 5-500 $\mathrm{ms}$ respectively.

Packets that are sent from the sender site generally have the same interval time and as they generated. They are transmitted to the real network through some different routes as they achieved. If a packet is not received up to the time of its playout, it is considered a lost packet. However, delay variation (jitter) constitutes more for the packet loss at the receiver site. Delay variation occurs because of traffic changes on the internet that automatically impact the transmitting of packetized data samples end to end.

To minimize the effect of variable network delays which is constitute to the packet loss, buffering delay at the receiver will smooth the jitter to the appropriate times. The use of a buffer time to generate a queuing time of packet as they received at the receiver will compensate the fluctuating end to end delays and variable network delays. Generally the larger the jitter, the bigger the buffer delay time inserted to the playout time at receiver will be. Unfortunately this additional delay will impair the human understanding and the QoS, whereas the adjustment to the low playout times will cause some packets to arrive too late and also it affect the

\footnotetext{
*E-MAIL: aasman2888@yahoo.com, aasman2888@gmail.com
}

perceived QoS. Therefore the buffering delay has to tradeoff between the loss of information and the determining of the waiting time to playout.

Some investigations considering the buffering delay have been published in few decades to present the determination of the buffer delay time to produce the scheduled time to playout arrived packet at the receiver site [1-3]. Some investigations have applied the fixed buffering delay time which used the fixed method of determination to fix buffer size of delay time. On the other hand, some investigations have presented the adaptive method. This method performs continuous estimation of the network delays and dynamically arranges the playout delay at the beginning of each talkspurt. The arrangement is applied on the first packet of the talkspurt where all packets in the same talkspurt are scheduled to play out at fixed intervals following the playout of the first packet.

The main objective of this investigation is to implement the presented investigation by Sakuray[4] regarding the VoIP communication to the streaming file transfer H.323 format for video compression over VLAN (Virtual LAN) using Network Analyzer for various Network behaviour. In addition, the playout algorithm showed that it can be implemented to the receiver site to reduce packet loss due to the effect of Jitter and the arrival time of each packet.

\section{PROPERTIES OF PACKET}

\section{A. CHUNK}

The structure of a packet depends on both the type of packet and the protocol and normally a packet has a header and a payload. The header keeps overhead information about the packet, the service and other transmission-related things. For 


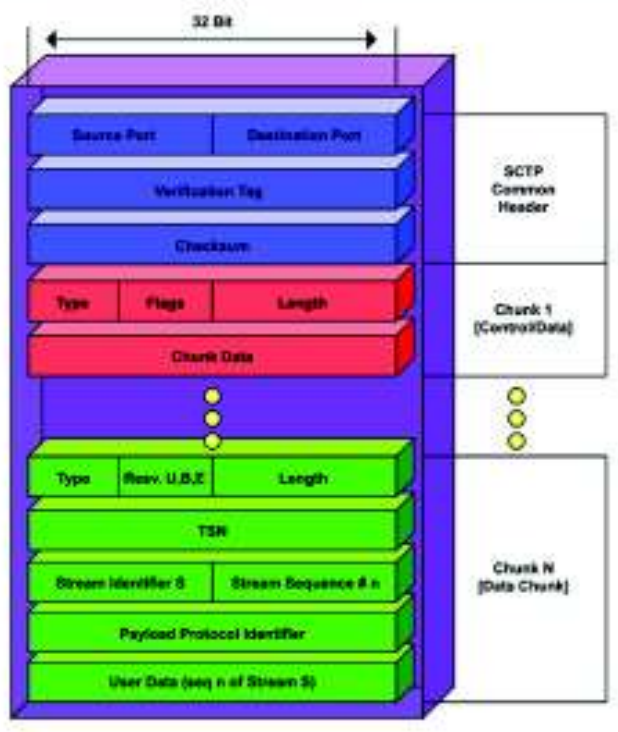

FIG. 1: SCTP packet data form

example, an IP packet includes: the source IP address, the destination IP address, the sequence number of the packets, the type of service, flags, etc.

\section{B. TALKSPURT}

In such Video and audio application like windows media player generates packets spaced at regular time intervals. The traffic generated by an audio and a video source is divided into periods of activity, called talkspurts, and periods of silence. The period of activity is a certain period where the data packet both video and audio generated and transferred from the source to the entire destination through network. The beginning of talkspurt indicated from the end of silence period as well as the traffic is started to transfer.

\section{PLAYOUT ALGORITHM}

\section{A. THEORETICAL DETERMINATION OF BUFFERING DELAY}

In Video and audio applications generate data packet with intervals time $\Delta \mathrm{t}$ in an active periods or talkspurt. A packet is transmitted at instant $t_{i}$ and is received at instant ai and executed at instant $\mathrm{p}_{i}$, as shown in Figure 1, the i-th packet of talkspurt $\mathrm{k}$ is sent at time $t_{k}^{i}$, it arrives at the receiver at time $a_{k}^{i}$, and is held in the smoothing receiver's playout buffer until time, when it is played out. Inside a talkspurt, packets are equally spaced at the sender by time intervals of length $\Delta t$ in seconds.

In Figure 2, a dropped packet due to a late arrival is viewed by a dashed line. A packet is artificially dropped if it arrives

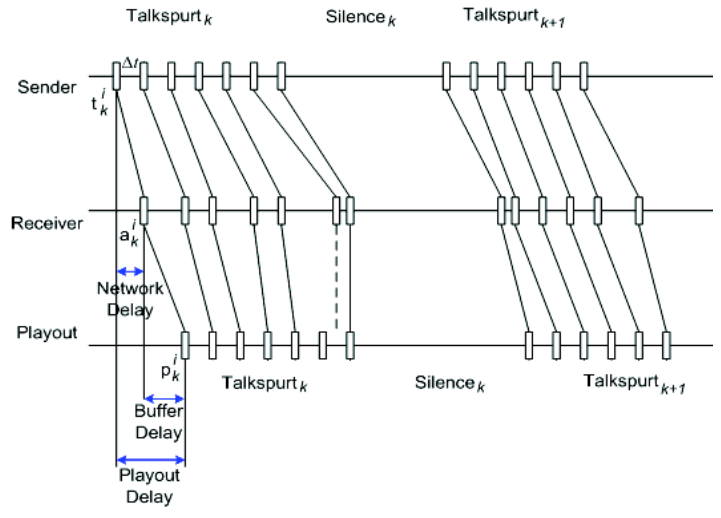

FIG. 2: Transmission of packets of a talkspurt

after its scheduled deadline . This loss can be reduced by increasing the amount of time that packets stay in the playout buffer. An efficient playout algorithm must take into account the trade-off between loss and delay in order to keep both parameters as low as possible.

Network latency $|\ell|$ is necessary to be determined because it will represent the time difference existing between the clock transmitter and receiver. The time $t_{i}$ will be reference at the receiver site, and will be replaced by $\left(\mathrm{t}_{i}-\ell\right)$ in such a way that if $\ell<0(\ell>0)$ the sender client will be advanced (delayed) compared to the receiver client and in case $\ell=0$. The time clock of both sender and receiver site will be synchronized by using NTP (Network time protocol) or using GPS (Global Position System).

The execution time period pi of the packets must respect the periodicity $\Delta \mathrm{t}$ applied by the sender site to transmit them, i.e., $p_{i}-p_{i-1}=\Delta \mathrm{t}$ for $\mathrm{i}=2, \ldots, \mathrm{n}$, or even $\mathrm{p}_{i}=\mathrm{p}_{1}+(\mathrm{i}-1) \Delta \mathrm{t}$. The execution time is scheduled based on the buffering delay time according to the playout algorithm which is calculated each buffering delay of $\mathrm{p}_{i}$ in one talkspurt.

Notice that packets that are loss with index $\mathrm{i}$ in a talkspurt is mathematically characterized when $\mathrm{p}_{i}<a_{i}$ or $\mathrm{p}_{i}-\left(\mathrm{t}_{i}-\ell\right) \leq \mathrm{L}$, in order to avoid the packet loss the algorithm has to meet the equation $\mathrm{p}_{i}-\mathrm{a}_{i} \geq 0$ and $\mathrm{p}_{i}-\left(\mathrm{t}_{i}-\ell\right) \leq \mathrm{L}$, for every $\mathrm{i} \in \mathrm{N}$. The first equation is known as playout restriction and the other is as latency restriction. This way, the playout delay $(\mathrm{Pd})$ of the packet with index $\mathrm{i}$ is given by $\mathrm{P}_{d i}=\mathrm{p}_{i}-\left(\mathrm{t}_{i}-\ell\right)$.

\section{B. DETERMINATION OF BUFFERING DELAY AND PLAYOUT DELAY}

In a talkspurt, if a packet with index $\mathrm{i}$ does not disturb the playout restriction, and the difference between the reception instant and sending instant overcomes the latency L, then the latency restriction is influenced by the packet with index $i$, no matter the buffer delay $\mathrm{T}$ used in talkspurt. In this session, consider a talkspurt with $\mathrm{n}$ number packets, having packet indexes given by $\mathrm{N}=1,2, \mathrm{n}$. the first result we present is a property referred to the latency restriction

The equation of Latency restriction $\mathrm{p}_{i}-\left(\mathrm{t}_{i}-\ell\right) \leq \mathrm{L}$, where 
$p_{i}=a_{i}+T+(i-1) \Delta t$ and $\mathrm{T}$ is an arbitrary buffer delay. Notice that $p_{i}-\left(t_{i}-\ell\right)=p_{i}-a_{i}+a_{1}-\left(t_{i}-\ell\right)$, besides $p_{i}-a_{i} \geq 0$ and $a_{i}-\left(t_{i}-\ell\right) \geq 0$, so $p_{i}-\left(t_{i}-\ell\right)>L$.

The network conditions impact the determination of the buffer delay. The previous property figures this fact, viewing under which conditions the network can determine unavoidable losses, independently from the choice of buffer delay. In this investigation, the conditions that deal to dimensioning the buffer delay have been tried to control by the algorithm. Without loss of generality of the results in order to consider that $a_{i}-\left(t_{i}-\ell\right)>0$, for every $i \in N$.

In a talkspurt where a buffer delay $\mathrm{T}$ is inserted, no packet will theoretically be lost, if and only if

$$
\underbrace{\max }_{i \in N}\left\{\delta_{i}-(i-1) \Delta t\right\} \leq T \leq \underbrace{\min }_{i \in N}\left\{\gamma_{i}-(i-1) \Delta t\right\}
$$

where $\gamma_{i}=\left(t_{i}-\ell\right)-a_{i}+L$ for every $i \in N$ and $\delta_{i}=a_{i}-a_{1}$

To prove the above equation, we can consider that if there is no packet loss at the talkspurt, this is equivalent to say that : $p_{i}-a_{i}=0$ and $p_{i}-\left(t_{i}-\ell>L\right)$ for every $i \in N \Leftrightarrow p_{i} \geq a_{i}$ and $p_{i}=\left(t_{i}-\ell\right)+L$ for every $i \in N \Leftrightarrow a_{i} \leq p_{i} \leq\left(t_{i}-\ell\right)+L$ for every $i \in N \Leftrightarrow a_{i} \leq a_{1}+T+(i-1) \Delta t \leq\left(t_{i}-\ell\right)+L$, for every

$\mathrm{i} \in N \Leftrightarrow \underbrace{\max }_{i \in N}\left\{\delta_{i}-(i-1) \Delta t\right\} \leq T \leq \underbrace{\min }_{i \in N}\left\{\gamma_{i}-(i-1) \Delta t\right\}$,

where $\gamma_{i}=\left(t_{i}-\ell\right)-a_{i}+L$, for every $i \in N$.

From the latter equation, we can note that $T_{\min }=$ $\underbrace{\max }_{i \in N} \delta_{i}-(i-1) \Delta t$ and,$T_{\max }=\underbrace{\max }_{i \in N} \delta_{i}-(i-1) \Delta t$ respectively, the minimum buffer delay and the maximum buffer delay, to which the packets loss is not verified.

The insertion of the maximum and minimum buffering delay will prove the playout algorithm to meet the trade-off minimum loss and the maximum latency. With $T_{\min }=$ $\underbrace{\max }_{i \in N} \delta_{i}-(i-1) \Delta t=\delta_{r}-(r-1 \Delta t)$, then:

$$
\begin{aligned}
p_{i} & =a_{1}+T_{\text {min }}+(i-1) \Delta t, \forall_{i} \in N \Leftrightarrow \\
p_{i} & =a_{1}+\delta_{r}-(r-1) \Delta t+(i-1) \Delta t, \forall_{i} \in N \Leftrightarrow \\
p_{i} & =a_{r}-(r-1) \Delta t, \forall_{i} \in N
\end{aligned}
$$

By considering that $\mathrm{i}=\mathrm{r}$, we have that $p_{r}=a_{r}$, the packet with index $\mathrm{r}$, which defines $\mathrm{T}_{m i n}$, is applied at the instant time of its reception. On the other hand, the part of equation with the maximum buffering delay will be applied to playout delay to get the bound of maximum latency, by considering $T_{\max }=$ $\underbrace{\max }_{i \in N} \gamma_{i}-(i-1) \Delta t$, then:

$$
\begin{aligned}
p_{i} & =a_{1}+T_{\max }+(i-1) \Delta t, \forall_{i} \in N \Leftrightarrow \\
p_{i} & =a_{1}+\gamma_{s}-(s-1) \Delta t+(i-1) \Delta t, \forall_{i} \in N \Leftrightarrow \\
p_{i} & =\left(t_{s}-\ell\right)+L-(s-1) \Delta t, \forall_{i} \in N
\end{aligned}
$$

Assuming $\mathrm{i}=\mathrm{s}$, we have that $p_{s}=\left(t_{s}-\ell\right)+L$, the packet with index $\mathrm{s}$, which defines $\mathrm{T}_{\max }$, is executed with maximum

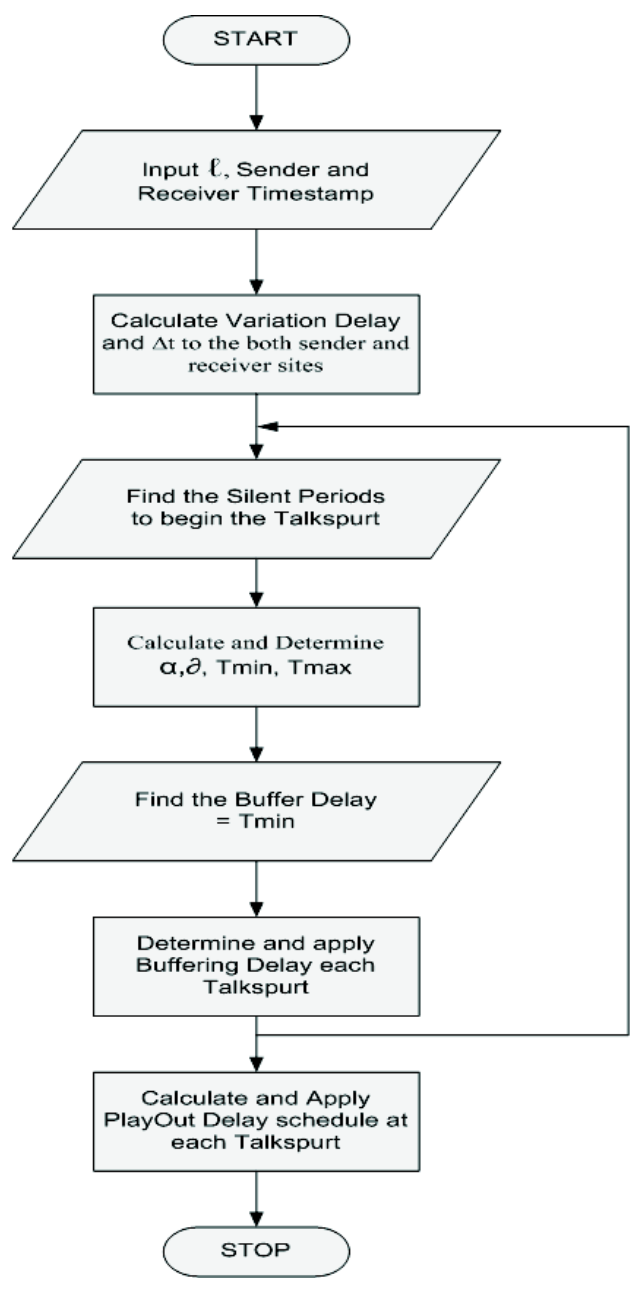

FIG. 3: Transmission of packets of a Determination of The Playout Algorithm Flowchart

latency. The insertion of the maximum and minimum buffering delay will prove the playout algorithm to meet the tradeoff minimum loss and the maximum latency.

\section{RESULT}

\section{A. PLAYOUT DELAY TIME}

The collected data sets are analyzed using the playout algorithm according to the formula and some equations to determine the buffering delay time and playout delay time. All result buffering delay and playout delay time have been underestimated for each talkspurt in one traces session of H.323 format. The results of the buffering delay estimation and playout algorithm will consider as packet loss if over or outside the playout time line. The next figure show the first talkspurt of H.323 video format and graphic of Playout algorithm result for one session from the data collection over VLAN network 


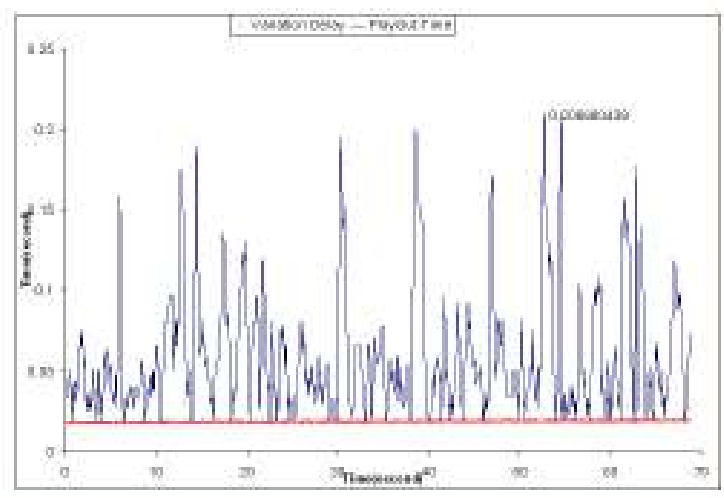

(a)

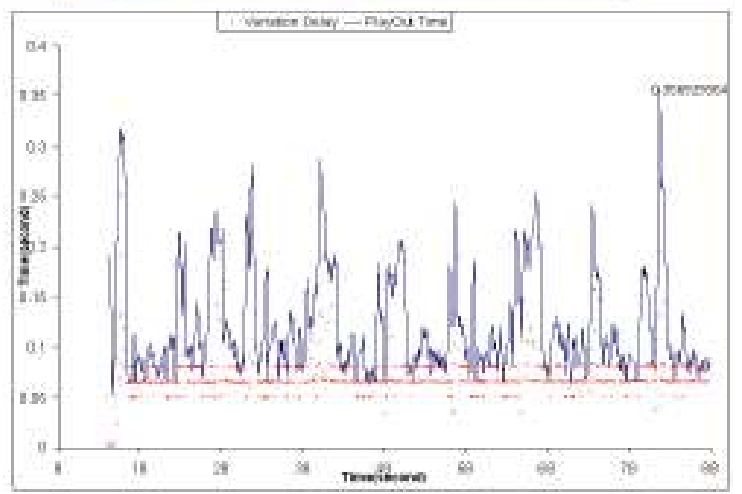

(c)

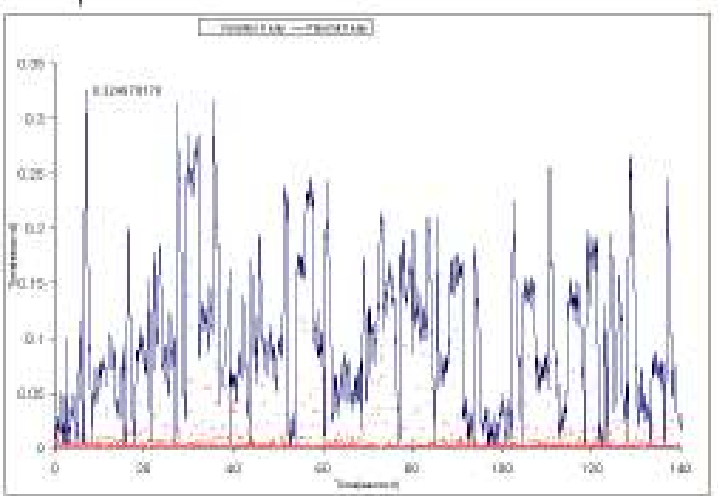

(b)

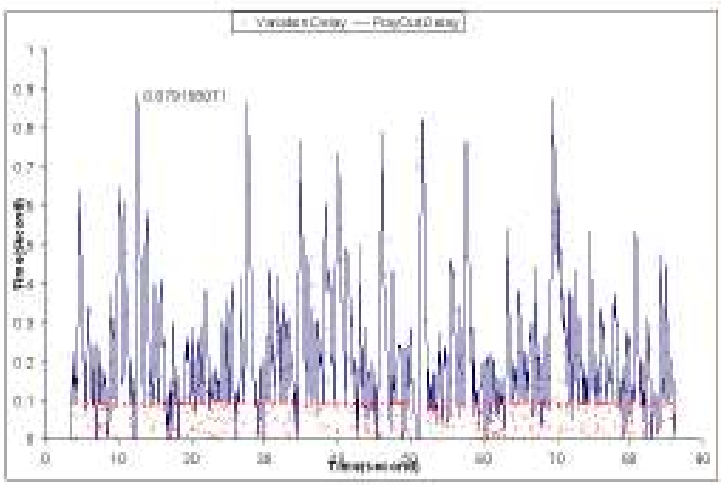

(d)

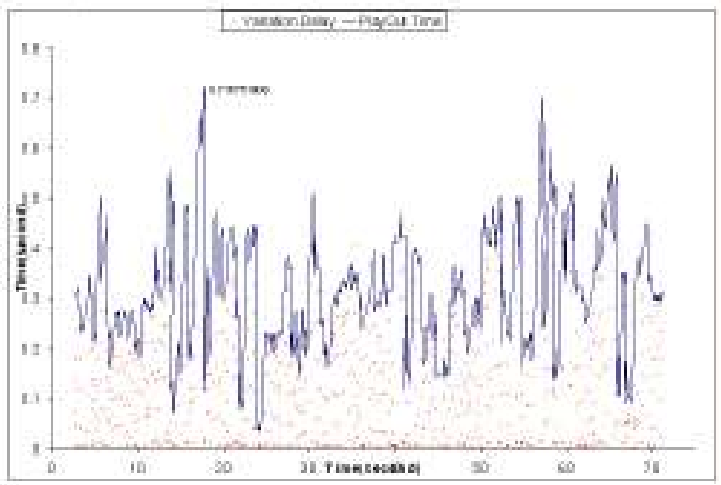

(e)

FIG. 4: PlayOut Algorithm in one session trace using H.323 video format with Load utilization (a). 5\%, (b).10\%, (c). 15\%, (d). 19\%, (e). $21 \%$.

by using Load Utilization $5 \%, 10 \%, 19 \%, 15 \%$, respectively.

\section{PACKET LOSS}

Various Jitter caused by network behavior will contribute to the packet loss in the receiver sites. The big packet loss in previous talkspurt will influence to determine the next playout time buffer so that it can increase the playout time buffering in the next talkspurt.

\section{CONCLUSION}

In streaming data file like H.323 video format, the increasing traffic load generation by using the network analyzer will 


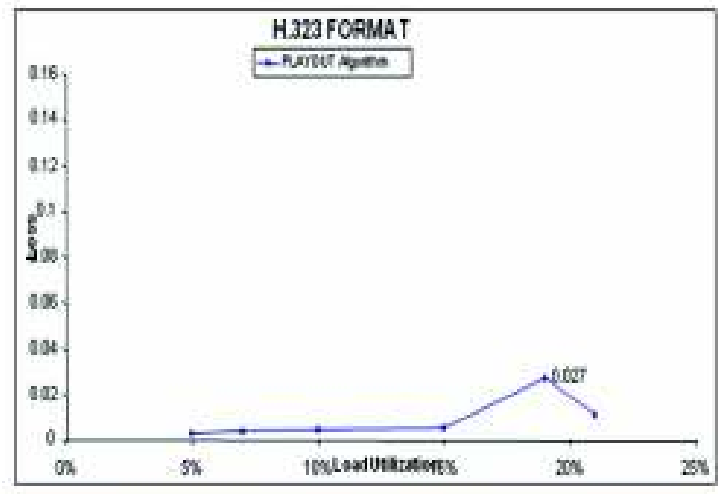

FIG. 5: Packet Loss vs Traffic Load utilization influence to the determination of the buffering delay time, playout delay time and packet loss. When the packet loss increases in the previous session, the playout time will be produced longer in the next session to reduce the packet loss. The packet loss of receiving packet in one session will be influenced by the increasing playout time buffering and our investigated playout algorithm also can keep low tradeoff for packet loss and buffering delay which is constitute under 5\% for $5 \%-21 \%$ traffic load utilization.
[1] DeLeon, P, Sreenan, J, Cormac, An Adaptive Predictor for Media Playout Buffering, IEEE Proceeding, 1999.

[2] Kalman, M, Steinbach, E, Girod, B, Adaptive Playout For RealTime Media Streaming, Information Systems Laboratory Department of Electrical Engineering Stanford University, IEEE Proceeding, 2002.

[3] Catherine Boutremans and Jean-Yves Le, Adaptive Joint Playout Buffer and FEC Adjustment for Internet Telephony, IEEE proceeding, 2003.

[4] Fbio Sakuray, Robinson S. V. Hoto and Leonardo S. Mendes, Analysis and Estimation of Playout Delay in VoIP Communi- cations, IJCSNS International Journal of Computer Science and Network Security, VOL.8 No.3, March (2008).

[5] Mohd Farhan Ngatman, Md Asri Ngadi, Johan M. Sharif, Comprehensive Study of Transmission Techniques for Reducing Packet Loss and Delay in Multimedia over IP, IJCSNS International Journal of Computer Science and Network Security, VOL.8 No.3, March (2008).

[6] Jesus Pinto and Kenneth J. Christensen, An Algorithm for Playout of Packet Voice based on Adaptive Adjustment of Talkspurt Silence Periods, IEEE proceeding, 1999. 